Research paper

\title{
Development of solid lipid nanoparticles as carriers for improving oral bioavailability of glibenclamide
}

\author{
L.M.D. Gonçalves ${ }^{\text {a }}$, F. Maestrelli ${ }^{\text {b,* }}$, L. Di Cesare Mannelli ${ }^{\text {c }}$, C. Ghelardini ${ }^{\text {c }}$, A.J. Almeida ${ }^{\text {a }}$, P. Mura ${ }^{\text {b }}$ \\ ${ }^{a}$ Research Institute for Medicines and Pharmaceutical Sciences (iMed.UL), Faculty of Pharmacy, University of Lisbon, Av. Prof. Gama Pinto, 1649-003 Lisboa, Portugal \\ ${ }^{b}$ Department of Chemistry, School of Sciences of Human Health, University of Florence, via Schiff 6, Sesto Fiorentino 50019, Florence, Italy \\ ${ }^{\mathrm{c}}$ Dept. of Neuroscience, Psychology, Drug Research and Child Health (Neurofarba)-Pharmacology and Toxicology Section, University of Florence, Florence, Italy
}

\section{A R T I C L E I N F O}

\section{Article history:}

Received 14 December 2015

Revised 9 February 2016

Accepted in revised form 19 February 2016

Available online 27 February 2016

\section{Keywords:}

Glibenclamide

Solid lipid nanoparticles

Stability

PEG coating

Antiglycemic effect

\begin{abstract}
A B S T R A C T
A solid lipid nanoparticle (SLN) formulation was developed with the aim of improving the oral bioavailability and the therapeutic effectiveness of glibenclamide (GLI), a poorly water-soluble drug used in the treatment of type 2 diabetes. The SLN was prepared using different lipid components (Precirol ${ }^{\circledR}$ and Compritol $^{\circledR}$ ) and preparation procedures. Precirol-based SLN, obtained with the emulsion of solvent evaporation technique gave the best results and was selected for drug loading. Addition of lecithin to the SLN core or PEG coating was effective in increasing the nanoparticles stability in simulated gastric solution. Both such formulations were stable after one month storage at $5 \pm 3{ }^{\circ} \mathrm{C}$, exhibited the absence of in vitro cytotoxicity, and presented a similar in vitro prolonged-release, reaching $100 \%$ release after $24 \mathrm{~h}$. The lecithin-containing GLI-loaded SLN formulation, selected for in vivo studies in virtue of its higher EE\% than the PEG-coated formulation (70.3\% vs 19.6\%), showed a significantly stronger hypoglycemic effect with respect to the drug alone, in terms of both shorter onset time and longer duration of the effect. These positive results indicated that the proposed SLN approach was successful in improving GLI oral bioavailability, confirming its potential as an effective delivery system for a suitable therapy of diabetes.
\end{abstract}

(c) 2016 Elsevier B.V. All rights reserved.

\section{Introduction}

Diabetes mellitus is one of the most common metabolic diseases, and $90 \%$ of diabetic patients in the world are affected by the non-insulin dependent Type 2 diabetes [22]. Hyperglycemia is a serious pathologic condition that can produce a variety of serious complications over a period of years, including neurological and cardiovascular damages. Sulfonylureas, the first widely used oral anti-hyperglycemic drugs, act as insulin secretagogues and trigger insulin release by inhibition of the ATP-sensitive potassium channels of the pancreatic beta-cells [14].

Glibenclamide (or glyburide), a second-generation sulfonylurea, is one of the most prescribed oral anti-hyperglycemic agents $[21,13]$. Due to its very poor water solubility and good permeability properties, GLI is classified as a BCS class II drug [55]. The very poor water-solubility of the drug is responsible for its limited and variable oral bioavailability [34], as well as for problems of nonbio-equivalence among its different commercial tablets [6].

* Corresponding author at: Chemistry Department, via Ugo Schiff, 6, 50019 Sesto Fiorentino, Florence, Italy.

E-mail address: francesca.maestrelli@unifi.it (F. Maestrelli).
Therefore, various approaches have been applied over the last years aimed at improving the solubility and dissolution properties of glibenclamide, including formation of binary [52,53] or ternary [11] solid dispersions in hydrophilic polymers, complexation with cyclodextrins $[18,10]$, combined use of cyclodextrins and polymers [61,12], formulation as liquid SMEDDS [3] or solid SMEDDS [33], or even as microparticles [35] or nanoparticles [58,47].

However, despite the efforts thus far, no completely satisfactory results have been still obtained and no glibenclamide products arising from these approaches are available in the market.

In recent years, the application of nanotechnology combined with the use of lipid-based formulations is receiving increasing interest as a promising strategy in drug delivery to enhance the oral bioavailability of hydrophobic drugs. As recently reviewed, these systems are able to increase the absorption of lipophilic drugs through the gastrointestinal tract by different mechanisms: (a) accelerating their dissolution process, facilitating the formation of solubilized phases, by particle size reduction to molecular level, thus yielding a solid-state solution within the carrier; (b) changing drug uptake, efflux and disposition, by altering the enterocytebased transport; and (c) enhancing the drug transport to the systemic circulation via the intestinal lymphatic system [23]. In 
particular, solid lipid nanoparticles (SLN) have emerged as an interesting and effective alternative to other particulate delivery systems such as nanoemulsions, micelles, liposomes, and polymeric nanoparticles $[16,30]$. It has been claimed that SLN should overcome some of the major disadvantages of other colloidal carriers, such as in particular the low stability and possible biotoxicity $[31,30]$, and offer several advantages such as high drug loading, protection from degradation and release modulation $[1,50,9,30]$. Many studies showed that SLN is actually able to prolong and/or control drug delivery, enhance its stability and improve its bioavailability $[27,49,60,38,19]$. In particular, the SLN effectiveness in improving the oral bioavailability of poorly-soluble drugs is mainly attributed to the nanoscale dimensions of the particles combined with the almost molecular dispersion of the drug molecules into the lipid carrier and the presence of surfactants, which strongly increases the drug gastrointestinal solubilization, and the in vivo absorption $[15,40,54]$.

However, in the development of SLN formulations, it has to be considered that many factors can affect their properties and the performance of the final product, such as the preparation method, the operative conditions, the nature of the materials used [16,30].

Based on all these premises, the aim of present work was the development of glibenclamide-loaded SLN able to improve its therapeutic efficacy and overcome the problems of poor and variable bioavailability. Different lipid components were examined and different preparation procedures were used for SLN production. The obtained formulations were characterized in terms of drug loading, particle size and zeta-potential, and solid-state properties. Stability and cytotoxicity studies were also performed on selected formulations. Finally, the best formulation was chosen for in vivo studies on diabetic rats, to evaluate its therapeutic efficacy in reducing the blood glucose levels.

\section{Materials and methods}

\subsection{Materials}

Glibenclamide (GLI) was a generous gift of Menarini (Italy). Glyceryl behenate (Compritol ${ }^{\circledR} 888$ ATO; COM) and glyceryl palmitostearate (Precirol ${ }^{\circledR}$ ATO5;PRE) were kindly provided by Gattefossé (Saint-Priest, Cedex, France); sodium deoxycholate (SDC), stearylamine (SA), polysorbate 20 (Tween 20), sodium dodecyl sulfate (SDS) and polyethylene glycol (average Mw 6000; PEG 6000) were obtained from Sigma-Aldrich. Soya lecithin (Lipoid S100) was a kind gift from Lipoid (Ludwigshafen, Germany). Purified water was obtained by reverse osmosis (Elix 3 Millipore, MD, USA). All other chemicals were at least of reagent grade and used as received.

\subsection{Preparation of SLN}

SLN was prepared using either the emulsification-solvent evaporation (ESE) or the hot high-shear homogenization $(\mathrm{HH})$ techniques, as previously described by Gaspar et al. [20] and Lopes et al. [27], respectively. Briefly, for the first method, the lipid (COM or PRE, $50 \mathrm{mg}$ ), alone or mixed with SA $(10 \mathrm{mg}$ ) was dissolved in dichloromethane, and then added dropwise to the aqueous phase containing Tween $20(5 \mathrm{mg} / \mathrm{mL})$ and SDC $(6 \mathrm{mg} / \mathrm{mL})$ and sonicated $(40 \mathrm{~W}, 2.5 \mathrm{~min}$, Branson Sonifier 250, USA). The obtained dispersion was homogenized for $3.5 \mathrm{~min}$ at $10,000 \mathrm{rpm}$ using a high shear homogenizer (Silverson L5M, UK). The dispersion obtained was then kept under magnetic stirring at $200 \mathrm{rpm}$ for $4 \mathrm{~h}$ at room temperature until dichloromethane complete evaporation.
According to the hot high-shear homogenization $(\mathrm{HH})$ method, the lipid (50 mg), with and without SA $(10 \mathrm{mg})$ was heated at about $80^{\circ} \mathrm{C}$ to obtain a clear solution. Afterward, purified water containing Tween $20(5 \mathrm{mg} / \mathrm{mL})$ and SDC $(6 \mathrm{mg} / \mathrm{mL})$, heated to the same temperature, was poured into the hot lipid phase and an emulsion was obtained by stirring $5 \mathrm{~min}$ at $10,000 \mathrm{rpm}$ with the high shear homogenizer (Silverson L5M, UK); finally the dispersion was cooled at $4{ }^{\circ} \mathrm{C}$. In the case of SLN incorporating GLI, the drug was added to the lipid phase.

Concerning stabilized SLN, empty and drug-loaded nanoparticles were prepared by the ESE technique, by adding lecithin to the lipid phase and/or molten PEG $6000(4.5 \% \mathrm{w} / \mathrm{w})$ in the lipid phase, and/or by coating SLN with PEG 6000 added (4.5\% w/w) to the aqueous phase with agitation for $4 \mathrm{~h}$ at room temperature.

\subsection{Measurement of particle size and zeta potential of SLN}

SLN mean diameter and polydispersity index (PI) were determined by quasi-elastic laser light scattering using a Malvern Zetasizer 1000HSA (Malvern Instruments; UK). The surface charge (zeta potential) was determined by laser Doppler anemometry using a Zetasizer 2000 (Malvern Instruments, UK). Samples were diluted appropriately for the measurements.

\subsection{Determination of drug entrapment efficiency (\%EE)}

The drug entrapment efficiency has been determined by both direct and indirect methods.

According to the direct method, the SLN dispersions, were first purified by size-exclusion chromatography in a PD-10 column (GE Healthcare, Germany) to remove the not incorporated drug. Then, $500 \mu \mathrm{L}$ of a solution of Triton ${ }^{\circledR} \mathrm{X}-100(10 \%), \mathrm{NaOH}(1 \mathrm{~N})$ and SDS (8\%) was added to the pre-treated colloidal dispersion and kept 2 min at $80^{\circ} \mathrm{C}$ to disrupt SLN; the free drug was then UV assayed at $300 \mathrm{~nm}$ using a microplate Reader FLUOstar Omega (BMG Labtech GmbH, Germany).

Entrapment efficiency (EE\%) of GLI in SLN was calculated according to the following equation:

$\% \mathrm{EE}=\left(W_{\text {entrapped drug }} / W_{\text {initial drug }}\right) \times 100$

where $W_{\text {initial }}$ drug is the drug amount initially used and $W_{\text {entrapped }}$ drug the drug amount entrapped into SLN.

According to the indirect method, the concentration of the not incorporated drug, present in the aqueous phase of the nanoparticle dispersion, was determined after separation from SLN by ultra filtration-centrifugation (centrifugal filters Amicon Ultra-4 with $100 \mathrm{kDa}$ molecular weight cut-off, Millipore, Germany). Briefly, $1 \mathrm{~mL}$ of GLI-loaded SLN dispersion was put into the upper chamber of the centrifuge filter, and then centrifuged at $4500 \mathrm{~g} 20 \mathrm{~min}$ at $4{ }^{\circ} \mathrm{C}$. The amount of free drug in the aqueous phase, collected in the outer chamber of the centrifuge filter, was then assayed as described above.

Entrapment efficiency (EE\%) of GLI in SLN was calculated according to the following equations:

$\% \mathrm{EE}=\left(W_{\text {initial drug }}-W_{\text {free drug }} / W_{\text {initial drug }}\right) \times 100$

where $W_{\text {free drug }}$ is the amount of free drug in the aqueous phase after separation of SLN.

\subsection{Stability studies}

Stability of SLN with time was evaluated both on the dispersions as such, stored at $5 \pm 3{ }^{\circ} \mathrm{C}$, or in the freeze-dried form. With this aim, after preparation, SLN formulations were divided into two aliquots of equal volume: one was frozen overnight and freeze-dried (Christ Alfa 1-4, Osterode am Harz, Germany), while 
the other one was kept at $5 \pm 3^{\circ} \mathrm{C}$. The stability of SLN in simulated gastrointestinal (GI) conditions was also evaluated.

\subsubsection{Stability of SLN dispersions}

Empty and drug-loaded SLN dispersions were stored at $5 \pm 3{ }^{\circ} \mathrm{C}$ for at least one month and checked for mean particle diameter, PI, zeta potential and $\mathrm{EE} \%$.

\subsubsection{Effect of freeze-drying}

To evaluate the effect of freeze-drying SLN formulations were freeze-dried with and without cryoprotectant $(10 \% \mathrm{w} / \mathrm{v}$ sucrose or trehalose).

\subsubsection{Effect of GI conditions}

To check the stability of SLN into the GI tract, and verify the absence of possible aggregation phenomena which could modify the particle size of the nanoparticles, and thus negatively affect their in vivo performance, a simulated gastric solution at $\mathrm{pH} 1.2$, alone ( $\mathrm{HCl} 0.1 \mathrm{~N}, \mathrm{~S} 1)$ or with pepsin (S2), and a simulated intestinal solution at $\mathrm{pH} 6.8$ (phosphate buffer, S3) were prepared. Then, $1 \mathrm{~mL}$ of each SLN dispersion was diluted with $9 \mathrm{~mL}$ of each GI fluid and incubated at $37{ }^{\circ} \mathrm{C}$ during $4 \mathrm{~h}$. Particle size, PI and zeta potential were tested every $30 \mathrm{~min}$ and $\mathrm{EE} \%$ at the end of the test.

\subsection{HPLC determination of glibenclamide (GLI)}

The drug HPLC assay was carried out by a LaChrom Elite HPLC apparatus (Merck Hitachi, Darmstadt, Germany) equipped with a L-2130 pump, an autosampler unit, and a L2450 UV/visible dual wavelength detector. A BDS C18 $(4.6 \mathrm{~mm} \times 100 \mathrm{~mm})$ Hypersil $^{\circledR}$ column (Thermo Electron Co., Waltham, MA, USA) was used as stationary phase. The mobile phase consisted of a 45:55 (v/v) mixture of acetonitrile/pH 3.0 phosphate buffer and a constant flow rate of $1.0 \mathrm{~mL} / \mathrm{min}$ was used. The detection was carried out at $210 \mathrm{~nm}$, the injection volume was $20 \mu \mathrm{L}$ and the column temperature was $35^{\circ} \mathrm{C}$. For these conditions, GLI is eluted at a retention time of $7.3 \mathrm{~min}$. A calibration curve in the $1-10 \mathrm{mg} / \mathrm{L}$ concentration range was prepared. The method was validated performing repeated analyses of decreasing analyte amounts [17]. The limit of quantification (LOQ) and limit of detection (LOD) were $1.012 \mathrm{mg} / \mathrm{L}$ and $0.3036 \mathrm{mg} / \mathrm{L}$, respectively.

\subsection{Differential Scanning Calorimetry (DSC)}

Thermal curves of individual components and of lyophilized SLN were recorded using a DSC Q200 (TA Instruments, DE, USA). Accurately weighed samples (1-2 mg) were placed in $\mathrm{Al}$ pans, which were hermetically sealed, and heated at $10^{\circ} \mathrm{C} / \mathrm{min}$ from 20 to $200^{\circ} \mathrm{C}$ under nitrogen, against an empty reference pan.

\subsection{Transmission electron microscopy (TEM)}

The morphological characteristics of the nanoparticles were investigated by TEM analysis. The samples were stained with phosphotungstic acid at $2 \%(\mathrm{w} / \mathrm{v})$ during $2 \mathrm{~min}$, fixed on racks of copper covered by a membrane of carbon for observation, and then analyzed on a JEOL Microscopy (JEM 2010, Japan) at $120 \mathrm{kV}$ and the images were acquired through a Gatan Orius ${ }^{\mathrm{TM}}$ camera.

\subsection{In vitro cell viability studies}

The absence of cytotoxicity of the developed SLN formulations was assessed using the Caco- 2 cell line, according to the MTT (3-(4,5-dimethylthiazol-2-yl)-2,5-diphenyl-2H-tetrazolium bromide) method, as previously described, with some modifications [8]. The Caco-2 cells (colorectal adenocarcinoma human cell line,
ATCC ${ }^{\circledR}$ HTB- $37^{\mathrm{TM}}$ ) were obtained from the American Type Cell Culture Collection. Cells were seeded on a 96-well plate at a cell density of $2 \cdot 10^{5}$ cells $/ \mathrm{mL} 24 \mathrm{~h}$ before the test and incubated at $37^{\circ} \mathrm{C}$ and 5\% CO2 in a humidified atmosphere. Cells were then incubated with both empty and GLI-loaded $(250 \mu \mathrm{g} / \mathrm{mL})$ SLN in RPMI 1640 culture medium (Gibco Invitrogen, Thermo Fisher Scientific, UK). SDS $(250 \mu \mathrm{g} / \mathrm{mL})$ was used as positive control, and the culture medium as negative control. After $24 \mathrm{~h}$, the nanoparticles dispersions (for test cells) or the culture medium (for negative control cells) or the SDS solution (for positive control cells) were removed and replaced with fresh medium. The cells were added $(200 \mu \mathrm{L}$ per well) with the MTT dye solution $(5 \mathrm{mg} / \mathrm{mL}$ ) and incubated $3 \mathrm{~h}$ at $37^{\circ} \mathrm{C}$. The MTT is converted, by living cells, into a dark, water insoluble, blue formazan product. After the incubation time, the MTT-containing medium was completely removed and the intracellular formazan crystals were dissolved and extracted with dimethylsulfoxide. After $15 \mathrm{~min}$ at room temperature, the absorbance of the extracted solution was measured at $570 \mathrm{~nm}$ using a microplate reader (Infinite M200, Tecan, Austria). The relative cell viability was determined by the following equation:

Cell viability $(\%$ of control $)=\mathrm{Abs}_{\text {sample }} / \mathrm{Abs}_{\text {control }} \times 100$

where $A b s_{\text {sample }}$ is the value obtained for cells treated with SLN or SDS and $\mathrm{Abs}_{\text {control }}$ the value obtained for cells incubated with the culture medium alone.

Each dosage was performed in triplicate, and each experiment was repeated at least 3 times.

\subsection{In vitro release studies}

The release studies were performed by a reverse dialysis bag technique [26,28]. Briefly, $1 \mathrm{mg}$ of drug as SLN dispersion, was placed in a vessel containing $250 \mathrm{~mL}$ of $\mathrm{pH} 7.4$ buffer at $37^{\circ} \mathrm{C}$ and magnetically stirred, where different dialysis sacs (Sigma Chemical Co, St. Louis, MO, 12,000 cut-off) containing $3 \mathrm{~mL}$ of $\mathrm{pH}$ 7.4 buffer were previously placed. At time intervals, dialysis sacs were removed and the drug concentration in solution was assayed by HPLC as described above. Each experiment ( $24 \mathrm{~h}$ ) was carried out in triplicate and the average values \pm S.D. were calculated.

\subsection{In vivo studies}

Clinically normal male Sprague-Dawley rats (Harlan, Varese, Italy), weighing approximately 280-300 g at the beginning of the experimental procedure, were used. Animals were housed in CeSAL (Centro Stabulazione Animali da Laboratorio, University of Florence) and acclimatized at least one week before their use. Two rats were housed per cage (size $26 \times 41 \mathrm{~cm}$ ); the animals were kept at $23 \pm 1{ }^{\circ} \mathrm{C}$ with a $12 \mathrm{~h} \mathrm{light/dark}$ cycle, fed with standard laboratory diet and tap water ad libitum. All animal manipulations were carried out according to the European Community guidelines for animal care (DL 116/92, Application of European Communities Council Directive of 24/11/1986 (86/609/EEC). The ethical policy of Florence University complies with the NIH Guide for the Care and Use of Laboratory Animals (US National Institutes of Health, Publication N. 85-23, revised 1996; University of Florence assurance number: A5278-01). Formal approval to conduct the experiments was obtained from the Animal Subjects Review Board of Florence University. Experiments involving animals have been reported according to ARRIVE guidelines [25]. All efforts were made to minimize animal suffering and reduce the number of animals used.

To induce diabetes mellitus to the rats, after blood collection for baseline glucose determination, they were subjected to a single intravenous tail vein injection of streptozotocin $(\mathrm{STZ}, 30 \mathrm{mg} / \mathrm{kg}$ 
body weight) (Sigma-Aldrich, Italy) dissolved in isotonic saline [57]. Age-matched control rats were injected with an equal volume of saline. On day 4 after STZ administration, the blood glucose level was measured (glucometer Accu-Chek Aviva, USA) and rats with blood glucose level $>200 \mathrm{mg} / \mathrm{dL}$ were considered diabetic and selected for experimentation. The diabetic rats were then randomly divided into three groups, each of six animals.

GLI, as such or formulated as SLN, at a dose of $5 \mathrm{mg} \mathrm{kg}^{-1}$ body weight was dispersed in water and orally administered to rats by gavage [36]. Control animals received the vehicle alone. Blood glucose levels were measured before and after (1, 2, 4, 6, 8 and $24 \mathrm{~h}$ ) drug administration, by collecting the blood samples from the tail vein of the animals. Results are expressed as mean \pm SEM. All data were analyzed by ANOVA (one-way analysis of variance) followed by a Bonferroni's significant difference procedure, used as post hoc comparison. Data were analyzed using the "Origin 8.1" software. Differences were considered statistically significant when $P$ values were $<0.05$.

\subsection{Statistical analysis}

Each batch was produced in three independent experiments and all the analyses were performed in triplicate. Results are expressed $(n=3)$ as mean \pm standard deviation (SD). Statistical evaluation of the data was performed by ANOVA (one-way analysis of variance) using the GraphPad Prism, version 4.0 program. Differences were considered statistically significant when $P$ values were $<0.05$.

\section{Results and discussion}

\subsection{Development of SLN formulation as carrier for glibenclamide (GLI)}

\subsubsection{Influence of the preparation method and of the type of lipid components}

Two different preparation procedures were used for SLN production i.e. the emulsification-solvent evaporation (ESE) and the Hot High-shear homogenization $(\mathrm{HH})$ techniques. Moreover, the influence of the type of lipid component type (COM or PRE), and of the presence or not of the positively charged SA were also investigated, while the aqueous phase was kept constant and based on a mixture of Tween $20(5 \mathrm{mg} / \mathrm{mL})$ and SDC $(6 \mathrm{mg} / \mathrm{mL})$ as co-surfactants. The obtained SLN formulations were characterized and compared in terms of mean particle dimensions, polydispersity index and zeta potential. The results of these studies, summarized in Table 1, showed that for all the examined formulations, the ESE method resulted in significantly smaller particles $(P<0.05)$, and higher homogeneity of the dispersion (as proved by the lower values of PI). However, the relative influence of the preparation method was found to be dependent from the SLN composition. In fact, SLN based on COM as lipid phase, alone

\section{Table 1}

Mean size, polydispersity index (PI) and zeta potential ( $\zeta$ ) of empty SLN made of Compritol (COM) or Precirol (PRE), containing or not stearylamine (SA), prepared by hot-homogenization $(\mathrm{HH})$ and emulsification-solvent evaporation (ESE).

\begin{tabular}{llllll}
\hline Batch & $\begin{array}{l}\text { Lipid } \\
\text { component }\end{array}$ & $\begin{array}{l}\text { Preparation } \\
\text { method }\end{array}$ & $\begin{array}{l}\text { Size } \\
(\mathrm{nm})\end{array}$ & PI & $\zeta(\mathrm{mV})$ \\
\hline SLN1a & COM & HH & $>1000$ & $0.84 \pm 0.21$ & $-44 \pm 2$ \\
SLN1b & COM & ESE & $330 \pm 10$ & $0.39 \pm 0.03$ & $-38 \pm 2$ \\
SLN2a & COM+SA & HH & $>1000$ & $0.59 \pm 0.07$ & $+14 \pm 1$ \\
SLN2b & COM+SA & ESE & Not obtained & \\
SLN3a & PRE & HH & $222 \pm 25$ & $0.63 \pm 0.17$ & $-38 \pm 2$ \\
SLN3b & PRE & ESE & $129 \pm 1$ & $0.23 \pm 0.06$ & $-34 \pm 3$ \\
SLN4a & PRE+SA & HH & $186 \pm 5$ & $0.39 \pm 0.04$ & $+8 \pm 1$ \\
SLN4b & PRE+SA & ESE & $159 \pm 6$ & $0.22 \pm 0.01$ & $+11 \pm 1$ \\
\hline
\end{tabular}

(SLN1) or in the presence of SA (SLN2), did not give particles in the desired submicron size and PI range with both the preparation methods, even though those obtained with the ESE method presented a mean size significantly smaller $(P>0.05)$ than those obtained with the HH method. On the contrary, SLN containing PRE, alone (SLN3) or with SA (SLN4), gave rise to good results in terms of mean size and PI with both methods, while confirming the better effectiveness of the ESE method. The addition of SA did not result in any significant reduction of the particle dimensions or improvement of their homogeneity, while it caused a marked decrease in the zeta potential, whose values, around $+10 \mathrm{mV}$, were considered not enough for allowing a good SLN stability and avoiding aggregation phenomena. Therefore, based on such findings, SLN based on PRE alone and prepared with the ESE technique (SLN3b) were selected to continue the study.

\subsubsection{GLI incorporation}

Preliminary solubility studies of GLI in PRE, performed before proceeding to the preparation of GLI-loaded PRE-based SLN, indicated that the drug solubility in the molten lipid was $2 \%(w / w)$, which should represent the highest amount of drug incorporable into the lipid. A series of SLN were prepared by adding increasing drug amounts (from 0.25 up to $2 \mathrm{mg}$ ) to the molten lipid $(50 \mathrm{mg})$. As a confirmation of the preliminary solubility studies, the drug entrapment efficiency increased with increasing its concentration up to $1 \mathrm{mg}$, reaching about $82 \%$, and then decreased, being exceeding the saturation solubility in the molten PRE. Moreover, a good agreement between EE\% data obtained by the direct and indirect methods has been found, thus confirming the reliability of the obtained results. Finally, $1 \mathrm{mg}$ GLI loading (SLN3bL) did not give rise to appreciable variations of particle size, PI and surface charge of SLN, as shown in Table 2.

\subsection{Stability studies of SLN}

\subsubsection{Stability of SLN dispersions}

The stability of the SLN formulations to maintain their physicochemical properties in terms of particle mean diameter, PI surface charge and drug entrapment was assessed after 1 month storage at $5 \pm 3{ }^{\circ} \mathrm{C}$. No significant changes $(P>0.05)$ were observed in mean size, PI and zeta potential values of empty or GLI-loaded SLN dispersions at the end of the storage period (Table 2). Also the entrapment efficiency remained almost constant, around $80 \%$, under these experimental conditions.

\subsubsection{Effect of freeze-drying of SLN}

Lyophilization has proved to be an effective way for enhancing the chemical and physical stability of SLN formulations over prolonged periods of time. However, freezing of the sample might cause problems of possible structural and/or functional damages of the system, and/or subsequent difficulties in sample resolubilization, due to particle aggregation phenomena [30].

The usefulness of addition of cryoprotectants in improving the quality of lyophilizates has been extensively investigated, and they proved to be able to decrease particle aggregation phenomena and obtain an easier re-dispersion of the freeze-dried product $[46,27]$. Therefore, to estimate the effect of the presence and type of cryoprotectant, empty and GLI-loaded SLN formulations were freeze-dried with and without sucrose or trehalose $(10 \% \mathrm{w} / \mathrm{v})$.

3.2.2.1. DSC study of freeze-dried SLN. Fig. 1 shows the thermal curves of pure GLI, PRE and cryoprotectants and of empty and drug-loaded lyophilized SLN, containing or not the cryoprotectant. The DSC curve of GLI presented a flat profile, with a sharp endothermal peak at $175.87^{\circ} \mathrm{C}\left(\Delta H_{\text {fus }} 107.15 \mathrm{~J} / \mathrm{g}\right)$, typical of its crystalline anhydrous nature. Similarly, the thermal curve of PRE 
Table 2

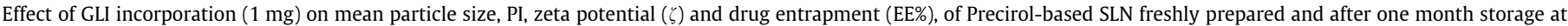
$5 \pm 3{ }^{\circ} \mathrm{C}($ mean $\pm \mathrm{SD}, n=3)$.

\begin{tabular}{|c|c|c|c|c|c|c|c|c|}
\hline \multirow[b]{3}{*}{ Sample } & \multicolumn{8}{|l|}{ Day } \\
\hline & 0 & 30 & 0 & 30 & 0 & 30 & 0 & 30 \\
\hline & \multicolumn{2}{|l|}{ Size $(\mathrm{nm})$} & \multicolumn{2}{|l|}{ PI } & \multicolumn{2}{|l|}{$\zeta(\mathrm{mV})$} & \multicolumn{2}{|l|}{$\mathrm{EE}(\%)$} \\
\hline SLN3b & $129.1 \pm 15.2$ & $128.2 \pm 7.1$ & $0.23 \pm 0.06$ & $0.28 \pm 0.07$ & $-34 \pm 3$ & $-41 \pm 3$ & 1 & 1 \\
\hline SLN3bL & $104.1 \pm 12.3$ & $112.1 \pm 13.2$ & $0.20 \pm 0.05$ & $0.33 \pm 0.08$ & $-35 \pm 3$ & $-43 \pm 3$ & $81 \pm 3$ & $80 \pm 5$ \\
\hline
\end{tabular}

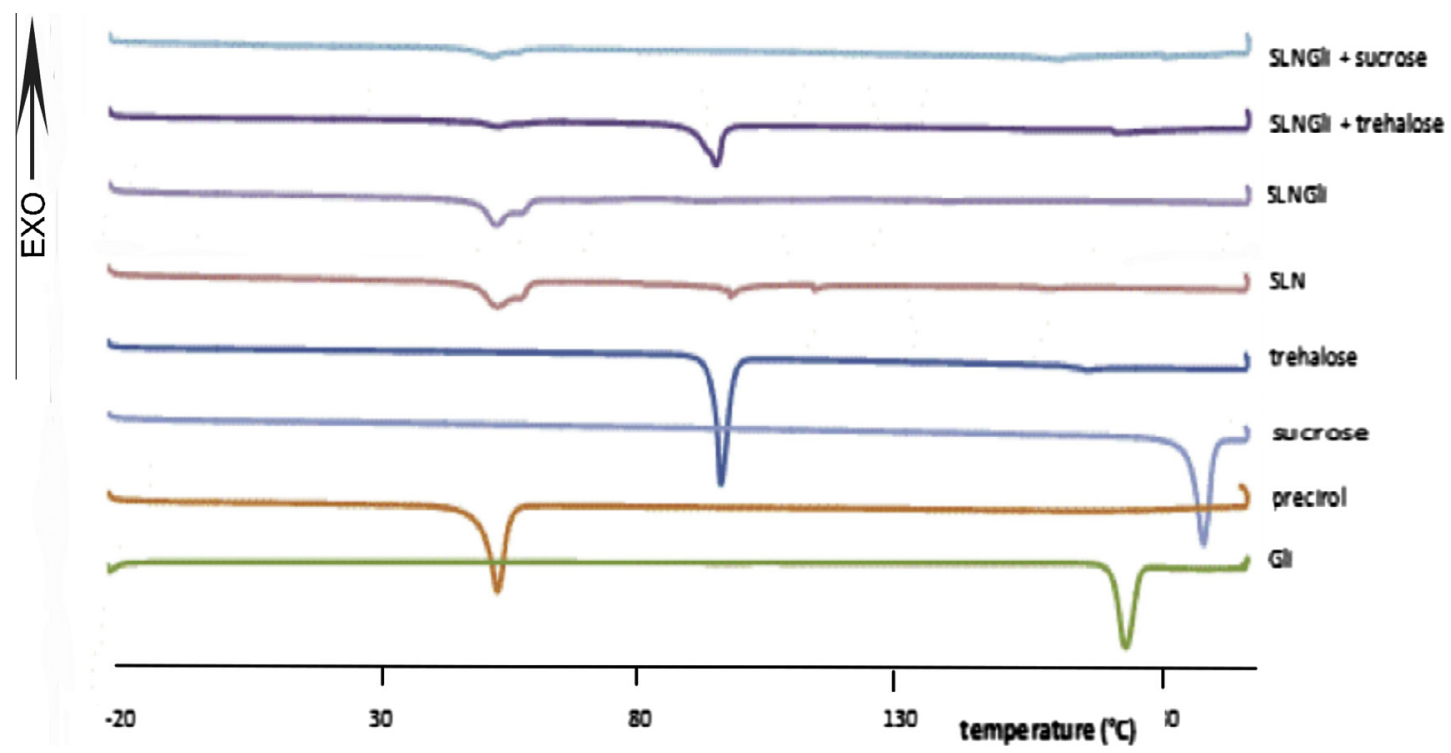

Fig. 1. DSC curves of pure components and of freeze-dried empty or GLI-loaded SLN, in the presence or not of sucrose or trehalose as cryoprotectants.

was characterized by an intense melting peak at $55.00^{\circ} \mathrm{C}\left(\Delta H_{\text {fus }}\right.$ $147.50 \mathrm{~J} / \mathrm{g}$ ). The melting peak of GLI disappeared in all the SLN samples, due to its amorphization or the almost molecular dispersion into the lipid matrix. In fact, the absence of the melting event of a crystalline drug in a lipid mixture is assumed as the effect of its solubilization in the lipidic mixture and/or its amorphization $[35,43]$. On the contrary, the melting peak of PRE was clearly detectable both in empty and in GLI-loaded SLN, even though it appeared clearly reduced in intensity, indicating a loss of crystallinity with respect to the pure lipid. The reduced crystallinity of PRE can be attributed to both the mixing of the formulation components and the SLN preparation method [7,48].

No appreciable variations were observed in the SLN thermal profile after drug incorporation. The same is true for the thermal curves of SLN containing the cryoprotectant, where a further reduction of intensity of the PRE melting peak was observed in both empty and loaded nanoparticles. As for the effect of the type of cryoprotectant, sucrose appeared to be more effective than trehalose in stabilizing the SLN structure. In fact its colyophilization with the SLN components caused its amorphization, proved by the disappearance of its melting peak $\left(190.83^{\circ} \mathrm{C}\right)$, and index of its complete and homogeneous dispersion within the lipid matrix, and, consequently, of its good protective effect against possible recrystallization phenomena of the amorphous matrix. On the contrary, the trehalose melting peak $\left(98.01^{\circ} \mathrm{C}\right)$ was still present both in empty and in loaded SLN, suggesting that it is less prone to be effectively incorporated and intimately dispersed into the lipid matrix.

\subsubsection{Stability in gastrointestinal (GI) conditions}

The first barrier to cross after SLN oral administration is represented by the physicochemical environment of the GI tract, which can negatively affect their stability and represent an obstacle to their absorption [44]. In particular, particle size plays a crucial role in their GI uptake and their clearance by the reticulo-endothelial system and it is estimated that a particle size less than $300 \mathrm{~nm}$ is advisable for the intestinal transport [30]. Stability of SLN dispersions was studied in different simulated gastrointestinal solutions: $\mathrm{pH} 1.2$ gastric solution alone (S1) and with pepsin (S2) and $\mathrm{pH} 6.8$ intestinal solution (S3). As it can be observed in Fig. 2, the behavior of empty and GLI-loaded SLN dispersions was very similar: in acid environment the size of empty and loaded SLN growth rapidly, reaching a 600-700 $\mathrm{nm}$ mean diameter after only 10 min of exposition, and then stabilized in a range between 750 and $900 \mathrm{~nm}$ until the end of the test ( $120 \mathrm{~min}$ ). The low $\mathrm{pH}$ of the gastric medium was responsible for this effect, reasonably due to critical agglomeration phenomena caused by the strong reduction of the surface charge of the particles, with zeta potential values close to $0 \mathrm{mV}$. On the contrary, the intestinal conditions did not cause important variations in terms of SLN size, whose range holds stable between 200 and $300 \mathrm{~nm}$ during $4 \mathrm{~h}$ of exposition, in virtue of the negative values of zeta potential, ranged around $-35 \mathrm{mV}$.

\subsubsection{Improvement of SLN stability in gastrointestinal (GI) conditions}

In order to stabilize SLN dispersions in the acidic gastric conditions and prevent as far as possible aggregation phenomena, allowing the particles to maintain almost unchanged their nanodimensions until their arrival to the basic environment of the intestinal tract, some formulations changes were experimented, by testing the effectiveness of the addition of lecithin and/or coating with PEG 6000.

The positive influence of lecithin in improving the stability of SLN formulations in terms of particle size and PI has been described in the literature $[41,27,29]$. This amphiphilic substance 

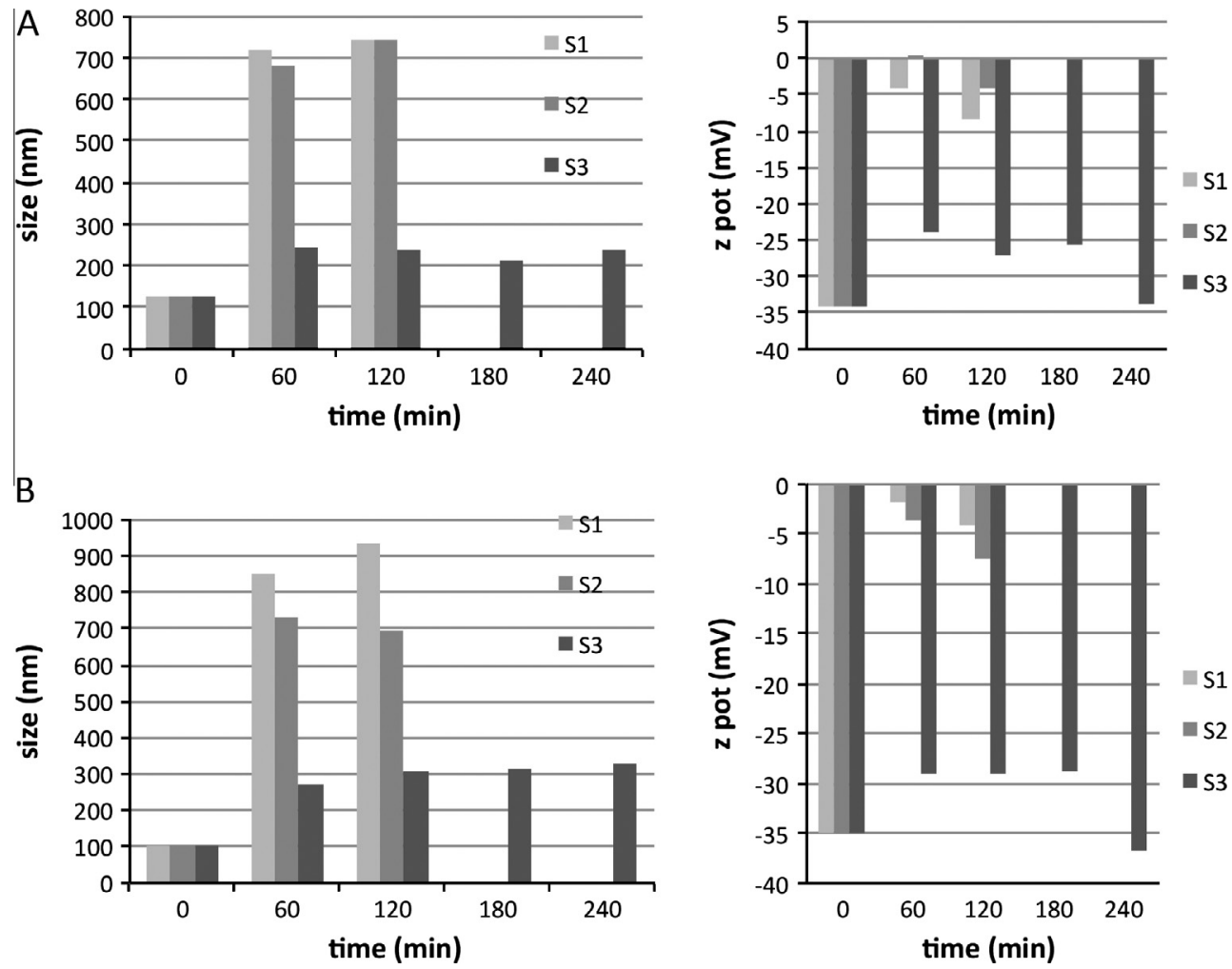

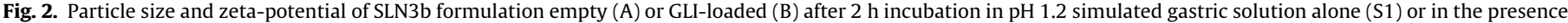
of pepsin (S2), and after $4 \mathrm{~h}$ in pH 6.8 simulated intestinal solution (S3). See Table 1 for SLN composition.

can reduce the interfacial tension and decrease interactions between the aqueous and organic phases, thus facilitating the droplets division during homogenization [56]. Moreover, the role of PEG coating in improving the stability of nanoparticles in digestive fluids $[51,24]$ and its stealth-effect on the prolongation of the colloidal vectors circulation [2] are well established. Therefore, different empty stabilized SLNs were initially prepared, as indicated in Table 3, by addition of lecithin into the lipid phase (SLN5) or by coating with PEG6000, in the absence (SLN6) or in the presence (SLN7) of lecithin; the possible effect of adding PEG6000 in the SLN core, in the absence (SLN8) and in the presence (SLN9) of lecithin, was also evaluated. The stabilized SLN formulations were then evaluated in terms of particle size, PI and surface charge, and compared to the empty reference formulation (SNL3b) after preparation and after $2 \mathrm{~h}$ incubation into the simulated gastric fluid (S1). As can be observed in Fig. 3, the reference formulation of SLN3bL showed a very low stability in gastric conditions, as indicated by a marked increase in particle size, and PI and reduction of zeta potential. These changes were also accompanied by a clear reduction of the entrapped drug amount; in fact, after $2 \mathrm{~h}$ in gastric conditions, the drug entrapment dropped to about $20 \%$. The better

Table 3

Composition and characteristics in terms of mean size, polydispersity index (PI) and zeta potential $(\zeta)$ of stabilized SLN formulations, empty and GLI-loaded (L)

\begin{tabular}{llllll}
\hline Batch & $\begin{array}{l}\text { Stabilizing } \\
\text { component }\end{array}$ & Coating & Size $(\mathrm{nm})$ & PI & $\zeta(\mathrm{mV})$ \\
\hline SLN5 & Lecithin & - & $112.2 \pm 6.1$ & $0.31 \pm 0.08$ & $-45 \pm 2$ \\
SLN6 & - & PEG 6000 & $183.1 \pm 3.2$ & $0.32 \pm 0.03$ & $-40 \pm 2$ \\
SLN7 & Lecithin & PEG 6000 & $140.2 \pm 5.4$ & $0.28 \pm 0.07$ & $-42 \pm 1$ \\
SLN8 & PEG6000 & - & $120.3 \pm 8.6$ & $0.23 \pm 0.04$ & $-42 \pm 1$ \\
SLN9 & PEG6000+ & - & $120.0 \pm 6.5$ & $0.25 \pm 0.17$ & $-37 \pm 2$ \\
& lecithin & & $100.2 \pm 4.2$ & $0.23 \pm 0.01$ & $-40 \pm 3$ \\
SLN5L & Lecithin & - & $105.1 \pm 2.9$ & $0.22 \pm 0.01$ & $-35 \pm 1$ \\
SLN7L & Lecithin & PEG 6000 & & & \\
\hline
\end{tabular}

results were obtained for formulations containing lecithin alone (SLN5), or in combination with PEG coating (SLN7), which both showed a clear stabilizing effect against the aggregation phenomena observed for the reference formulation, with a variation of the mean diameter less than $0.8 \%$ after $2 \mathrm{~h}$ in the simulated gastric medium. On the contrary, both addition of PEG6000 alone in the SLN core (SLN8), and PEG-coating in the absence of lecithin (SLN6) were not effective in stabilizing SLN in the gastric medium. Finally, the combined use of lecithin and PEG 6000 in the core of the nanoparticles (SLN9) was able to significantly reduce the particle size increase in SLN in acidic medium, but gave rise to a very poorly homogeneous dispersion, with a PI value around 0.8 . Therefore, formulations with lecithin alone (SLN5), or combined with the PEG 6000 coating (SLN7) were selected for drug loading with $1 \mathrm{mg}$ GLI (SLN5L and SLN7L, respectively). The two stabilized GLI-loaded SLN formulations exhibited similar mean size values around $100 \mathrm{~nm}$ and low PI values, not significantly different from the reference loaded formulation without lecithin and PEG 6000 (SLN3bL). Moreover, even though an increase in the mean particle dimension of both the stabilized SLN formulations was observed after $2 \mathrm{~h}$ in acidic medium, their final size did not overcome $160 \mu \mathrm{m}$, and a good homogeneity of the dispersions was kept, with PI values around 0.3 .

Stabilized SLN containing lecithin alone showed a good EE\% of $70.3 \pm 5.2$, while, unexpectedly, a clearly lower EE\% value $(19.6 \pm 4.8)$ was found for those with PEG coating, despite their similar dimensions and stability properties. The lower entrapment efficiency of SLN7L could be attributed to the possible solubilizing and wetting effects of PEG toward GLI $[4,11]$, which could produce a hydrophilic environment around the drug, thus reducing its affinity for the SLN lipid core, and/or favoring some drug losing during the SLN formation. It was also checked that the $\mathrm{EE} \%$ values of both these SLN formulations remained almost unchanged after $2 \mathrm{~h}$ in acidic medium. 

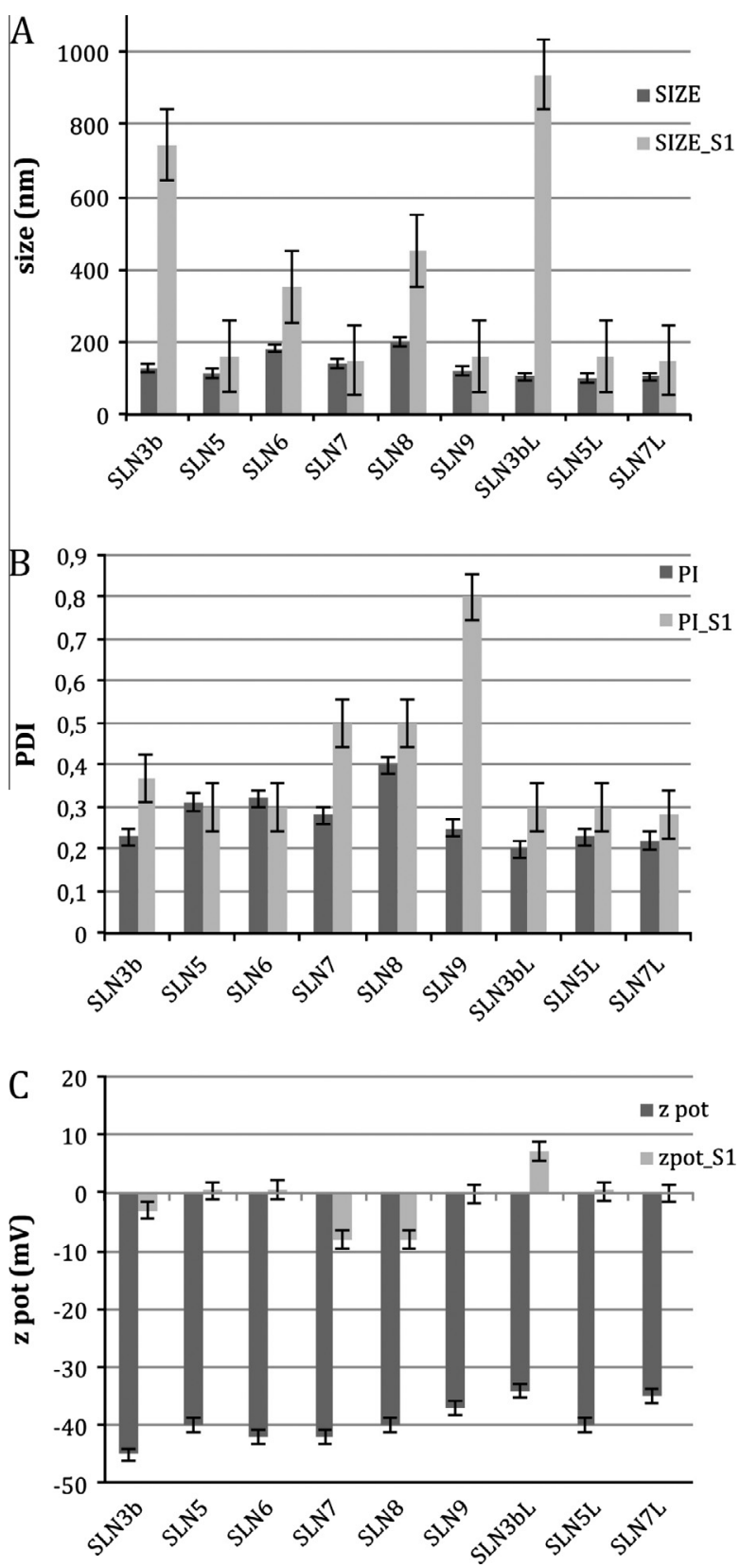

Fig. 3. Particle size (A), polydispersity index (B) and zeta-potential (C) of the different SLN formulations before or after $2 \mathrm{~h}$ incubation in $\mathrm{pH} 1.2$ simulated gastric solution (S1). See Tables 1 and 3 for SLN composition.

\subsection{Cell viability studies}

The cytotoxicity of the selected stabilized SLN formulations was assessed on Caco-2 cells, according to the MTT assay. Fig. 4 shows the percent of survival of Caco-2 cells at 24 and $48 \mathrm{~h}$ after treatment with empty SLN (SLN3b, F1) and GLI-loaded SLN uncoated (SLN5L, F2) or PEG-coated (SLN7L, F3). The results clearly proved the absence of cytotoxicity effect for all the samples tested, with no significant variations in\% cell viability with respect to the negative control (pure culture medium), confirming that the developed SLN formulations are safe and highly biocompatible.

\subsection{In vitro release studies}

The formulations of SLN5L and SLN7L were selected for drug release studies. Amounts of formulations containing $1 \mathrm{mg}$ of drug

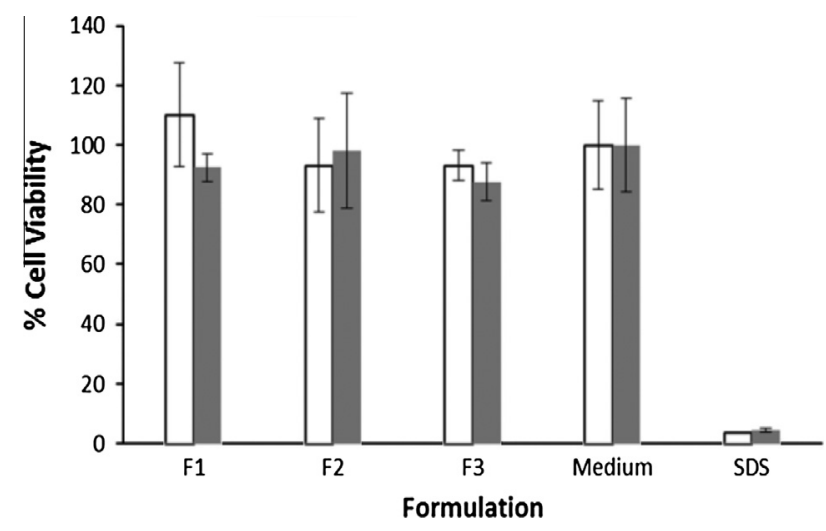

Fig. 4. Effect on cell viability of empty SLN (SLN3b) (F1) and GLI-loaded SLN uncoated (SLN5L, F2) or PEG-coated (SLN7L, F3) after 24 ( $\square$ ) and $48 \mathrm{~h} \mathrm{( \square )}$ exposition. See Tables 1 and 3 for SLN composition.

were tested by the dialysis bag method and the results are presented in Fig. 5. The dialysis of both SLN formulations showed a typical biphasic profile, characterized by an initial rapid release phase, where about $20 \%$ was released within the first $30 \mathrm{~min}$, followed by a second phase with a slower release rate, where about $100 \%$ release was achieved after $24 \mathrm{~h}$. No significant differences were observed between the two SLN formulations.

\subsection{TEM analysis}

TEM photographs of selected SLN batches (Fig. 6) demonstrated the actual formation of homogeneous nanosized round particles. Empty SLN5 particles (Fig. 6A) showed dimensions ranged between 100 and $200 \mathrm{~nm}$, thus supporting the results of dynamic light scattering analysis. The corresponding GLI-loaded SLN particles (SLN5L) evidenced similar results (Fig. 6B), confirming that drug loading did not modify shape and dimensions of the solid lipid nanoparticles.

\subsection{In vivo studies of anti-glycemic effect of GLI-loaded SLN}

The effectiveness of the developed SLN formulation in improving the anti-glycemic effect of GLI was investigated in vivo on male rats rendered diabetic by a single intravenous tail vein injection of STZ, in comparison with the simple aqueous dispersion of the drug, both orally administered by gavage $\left(5 \mathrm{mg} \mathrm{kg}^{-1}\right.$ body weight). The SLN5L formulation was selected for this study, in virtue of its

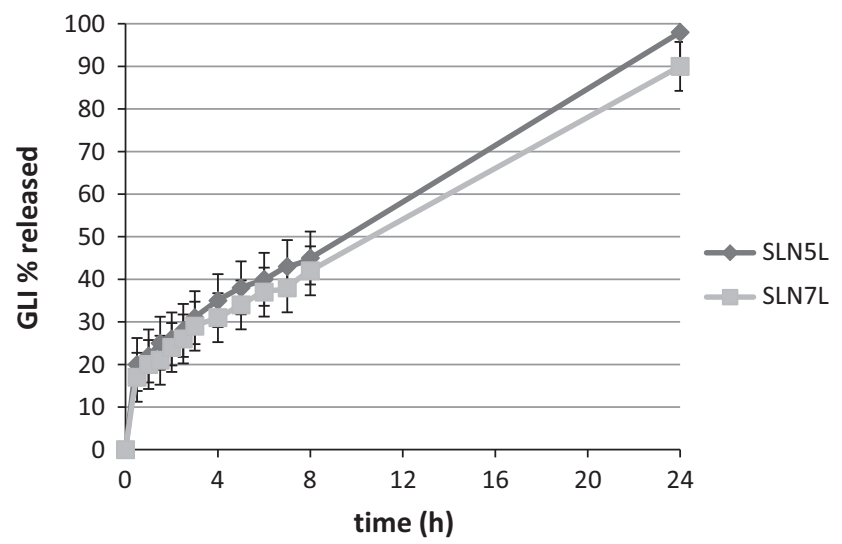

Fig. 5. Release profiles of glibenclamide from SLN5L $(\bullet)$ and SLN7L formulations. 

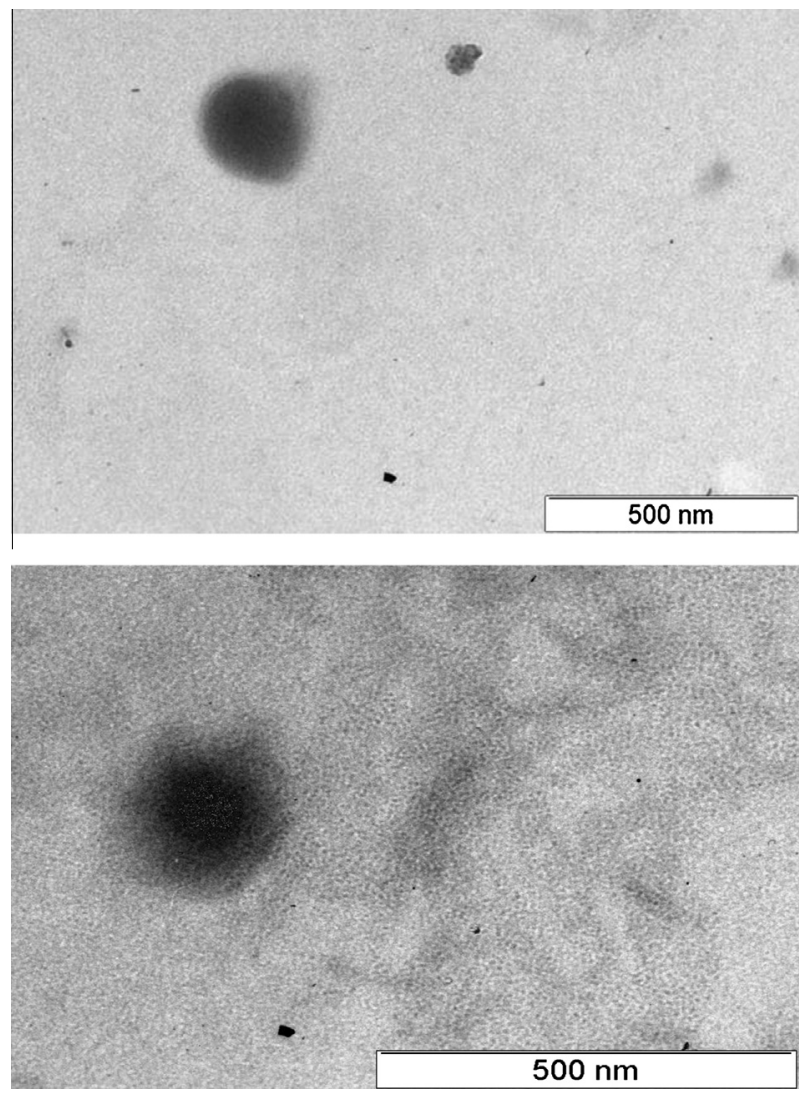

Fig. 6. TEM photographs of empty SLN (SLN5) (top) and GLI-loaded (SLN5L) (bottom). See Table 3 for SLN composition.

higher EE\% than the SLN7L (70.3\% vs $19.6 \%$ ). The results, collected in Table 4, showed that the reduction of the blood glucose level obtained after drug alone administration reached an acceptable value only after $4 \mathrm{~h}$ and then climbed rather quickly to the initial high levels, becoming no more significantly different from the pre-test value after $8 \mathrm{~h}$. In contrast, the administration of GLI formulated as SLN not only showed a more rapid onset of the blood glucose level reduction, which became significantly different from the pre-test just $1 \mathrm{~h}$ after administration, but also remained significantly lower than the pre-test value also after $8 \mathrm{~h}$. Moreover, the anti-glycemic effect obtained with the SLN formulation remained significantly higher than that given by drug alone from the first until to the eighth hour after drug administration, further confirming the higher therapeutic effectiveness of the GLI-SLN formulation.

Most of the previous reports about the use of different formulative strategies to improve the dissolution properties of GLI $[18,53,10,61,11,12,3,58,33,47]$ do not provide data about the actual consequent improvement of in vivo bioavailability and therapeutic efficacy of the drug from such formulations. On the other hand, an increase in GLI bioavailability has been reported for its solid dispersions in Gelucire or PEG 6000 with respect to a commercial formulation (Daonil tablets (Hoechst)), but without any effect on the duration of the antiglycemic action [52]. On the contrary, a prolonged effect was found from GLI solid lipid microparticles, but accompanied by a some decrease in the intensity of the antiglycemic effect with respect to the commercial formulation [35]. Therefore, no one of the previously reported formulative approaches was able to improve at the same time both the intensity and the duration of the drug therapeutic effect, as instead we obtained with the GLI SLN formulation.

The significant enhancement of GLI hypoglycemic effect was observed after its administration as SLN dispersion can be attributed to an improved drug absorption from the lipid-based carrier, even though the mechanisms underlying this effect still remain to be fully understood. In fact, the reasons for the improved oral bioavailability of drugs, obtained by lipid-based delivery systems, including SLN, as well as the question if the drugs formulated as SLN are absorbed as free drug or in the form of SLN have not been well clarified yet, even though different possible mechanisms have been proposed to provide an interpretation to the absorptionenhancing phenomenon. Among these, one of the most accepted is that SLN can facilitate the oral absorption of poorly-water soluble drugs by maintaining a solubilized state of the drugs in the GI tract, and by facilitating the formation of mixed micelles, promoting secretion of endogenous phospholipids and bile salts $[32,39]$. Besides, bioadhesion of SLNs to the gut wall seems to prolong the residence time of SLNs in the GI tract and enhance their intimate contact with epithelial membranes, which possibly contribute to enhance oral drug absorption [32,62]. The potential muco-penetrating ability of SLN is another aspect possibly responsible for the enhanced oral drug absorption [5,59]. Also the small particle size and the surface characteristics of the nanoparticles have an important role [42]. An additional reason for the improved drug absorption from SLN could be attributed to the enhancement of lymphatic delivery: lipids can stimulate lipoprotein formation and intestinal lymphatic lipid flux to increase the extent of lymphatic drug transport $[37,45]$.

\section{Conclusions}

A SLN formulation has been developed aimed to overcome the problems of poor and variable oral bioavailability of GLI and improve its therapeutic effectiveness. Preliminary studies performed on empty nanoparticles enabled selection of the best preparation technique (emulsion solvent evaporation) and the best lipid component (Precirol ${ }^{\circledR}$ ) to use for preparation of GLIloaded SLN with the desired nanosized dimension and high homogeneity. Addition of lecithin to the SLN core or PEG coating was effective in increasing the nanoparticles stability in simulated gastric environment, avoiding aggregation phenomena. Both the developed formulations were stable after one month storage at $5 \pm 3{ }^{\circ} \mathrm{C}$, exhibited the absence of cytotoxicity, and showed similar prolonged release profiles.

The lecithin-containing GLI-loaded SLN formulation was selected for in vivo studies, due to its higher EE\% than the PEGcoated formulation. The results of in vivo studies evidenced the significantly stronger hypoglycemic effect of such formulation,

Table 4

Blood glucose levels of diabetic rats after oral drug administration ( $5 \mathrm{mg} \mathrm{kg}^{-1}$ body weight) such as (GLI) or formulated as SLN (SLN5L).

\begin{tabular}{|c|c|c|c|c|c|c|c|}
\hline \multicolumn{8}{|c|}{ Blood glucose levels (mg/dL) } \\
\hline Treatment & Pretest & $1 \mathrm{~h}$ & $2 \mathrm{~h}$ & $4 \mathrm{~h}$ & $6 \mathrm{~h}$ & $8 \mathrm{~h}$ & $24 \mathrm{~h}$ \\
\hline $\mathrm{STZ}+\mathrm{GLI}$ & $340.6 \pm 24.6$ & $322.0 \pm 21.6$ & $193.0 \pm 18.0^{\wedge}$ & $120.3 \pm 11.6^{\wedge}$ & $197.0 \pm 11.2^{\wedge}$ & $299.9 \pm 22.5$ & $325.6 \pm 20.4$ \\
\hline STZ + SLN5L & $334.0 \pm 12.8$ & $261.0 \pm 18.1^{\wedge} . \S$ & $142.6 \pm 8.9^{\wedge} \S$ & $95.3 \pm 12.7^{\wedge} \S$ & $160.6 \pm 10.3^{\wedge}, \S$ & $255.6 \pm 25.3^{\wedge}, \S$ & $330.5 \pm 18.8$ \\
\hline
\end{tabular}

$P<0.05$ vs pretest of the same group.

$\S P<0.05$ vs STZ + GLI group. 
with respect to the drug alone, in terms of both shorter onset time and longer duration of the effect.

Even if more in-depth investigations will be needed to elucidate the effective mechanisms responsible for the improved drug absorption, these positive results indicated that the proposed SLN approach was successful in improving GLI oral bioavailability, showing its potential in obtaining a more effective GLI-based oral therapy of diabetes mellitus.

\section{References}

[1] A.J. Almeida, E. Souto, Solid lipid nanoparticles as a drug delivery system for peptides and proteins, Adv. Drug Deliv. Rev. 59 (2007) 478-490.

[2] Z. Amoozgar, Y. Yeo, Recent advances in stealth coating of nanoparticle drug delivery systems, Wiley Interdiscip Rev Nanomed Nanobiotechnol 4 (2012) 219-233.

[3] Y.G. Bachhav, V.B. Patravale, SMEDDS of gliburide: formulation in vitro evaluation, and stability studies, AAPS PharmSciTech. 10 (2) (2009) 482-487.

[4] G.V. Betageri, K.R. Makarla, Enhancement of dissolution of glyburide by solid dispersion and lyophilization techniques, Int. J. Pharm. 126 (1995) 155-160.

[5] I. Behrens, A.I. Pena, M.J. Alonso, T. Kissel, Comparative uptake studies of bioadhesive and non-bioadhesive nanoparticles in human intestinal cell lines and rats: the effect of mucus on particle adsorption and transport, Pharm. Res. 19 (2002) 1185-1193.

[6] H. Blume, S.L. Ali, M. Siewert, Pharmaceutical quality of glibenclamide products a multinational postmarket comparative study, Drug Dev. Ind. Pharm. 19 (1993) 2713-2741.

[7] H. Bunjes, T. Unruh, Characterization of lipid nanoparticles by differential scanning calorimetry, X-ray and neutron scattering, Adv. Drug Deliv. Rev. 59 (2007) 379-402.

[8] A. Cadete, L. Figueiredo, R. Lopes, C.C.R. Calado, A.J. Almeida, L.M.D. Gonçalves, Development and characterization of a new plasmid delivery system based on chitosan-sodium deoxycholate nanoparticles, Eur. J. Pharm. Sci. 45 (2012) 451-458.

[9] S. Chakraborty, D. Shukla, B. Mishra, S. Singh, Lipid - an emerging platform for oral delivery of drugs with poor bioavailability, Eur. J. Pharm. Biopharm. 73 (2009) 1-15.

[10] M. Cirri, F. Maestrelli, S. Furlanetto, P. Mura, Solid state characterization of gliburide-cyclodextrin co-ground products, J. Therm. Anal. Calorim. 77 (2004) 413-422.

[11] M. Cirri, M. Valleri, F. Maestrelli, G. Corti, P. Mura, Fast-dissolving tablets of gliburide based on ternary solid dispersions with PEG 6000 and surfactants, Drug Deliv. 14 (2007) 247-255.

[12] M. Cirri, M.F. Righi, F. Maestrelli, P. Mura, M. Valleri, Development of gliburide fast-dissolving tablets based on the combined use of cyclodextrins and polymers, Drug Dev. Ind. Pharm. 27 (2009) 1-10.

[13] S.W. Coppack, A.F. Lant, C.S. McIntosh, A.V. Rodgers, Pharmacokinetic and pharmacodynamic studies of glibenclamide in non-insulin dependent diabetes mellitus, Br. J. Clin. Pharmacol. 29 (1990) 673-684.

[14] S. Del Prato, N. Pulizzi, The place of sulfonylureas in the therapy for type 2 diabetes mellitus, Metabolism 55 (2005) 20-27.

[15] M. Durán-Lobato, L. Martín-Banderas, L. Gonçalves, M. Fernández-Arévalo, A.J Almeida, Comparative study of chitosan- and PEG-coated lipid and polymeric nanoparticles as oral delivery systems for cannabinoids, J. Nanopart. Res. (2015) 1761.

[16] A. Elgart, I. Cherniakov, Y. Aldouby, A.J. Domb, A. Hoffman, Lipospheres and pro-nano lipospheres for delivery of poorly water soluble compounds, Chem. Phys. Lip. 165 (2012) 438-453.

[17] J. Ermer, Validation in pharmaceutical analysis. Part I: an integrated approach, J. Pharm. Biomed. Anal. 24 (2001) 755-767.

[18] M.T. Esclusa-Díaz, J.J. Torres-Labandeira, M. Kata, J.L. Vila-Jato, Inclusion complexation of glibenclamide with 2-hydroxypropyl- $\beta$-cyclodextrin in solution and in solid state, Eur. J. Pharm. Sci. 1 (1994) 291-296.

[19] T. Fan, C. Chen, H. Guo, J. Xu, J. Zhang, X. Zhu, Y. Yang, Z. Zhou, L. Li, Y. Huang, Design and evaluation of solid lipid nanoparticles modified with peptide ligand for oral delivery of protein drugs, Eur. J. Pharm. Biopharm. 88 (2014) $518-528$.

[20] D.P. Gaspar, V. Faria, L. Gonçalves, P. Taboada, C. Remuñán-López, A.J. Almeida, Rifabutin-loaded solid lipid nanoparticles for inhaled antitubercular therapy: physicochemical and in vitro studies, Int. J. Pharm. 497 (2015) 199-209.

[21] L. Groop, E. Wahlin-Boll, K.J. Totterman, A. Melander, E.M. Tolppanen, F. Fyhrqvist, Pharmacokinetics and metabolic effects of glibenclamide and glipizide in type 2 diabetes, Eur. J. Clin. Pharmacol. 28 (1985) 697-704.

[22] H. Jun, Y.B. Hak, R.L. Byoung, S.K. Kwang, S.K. Young, W.L. Kwan, K. Hyun-man, Y. Ji-Won, Pathogenesis of non-insulin-dependent (type II) diabetes mellitus (NIDDM) - genetic predisposition and metabolic abnormalities, Adv. Drug Deliv. Rev. 35 (1999) 157-177.

[23] S. Kalepu, M. Manthina, V. Padavala, Oral lipid-based drug delivery systems an overview, Acta Pharm. Sin. B 3 (2013) 361-372.

[24] S. Kashanian, E. Rostami, PEG-stearate coated solid lipid nanoparticles as levothyroxine carriers for oral administration, J. Nanoparticle Res. 16 (2014) $1-10$
[25] C. Kilkenny, W.J. Browne, I.C. Cuthill, M. Emerson, D.G. Altman, Improving bioscience research reporting: the ARRIVE guidelines for reporting animal research, J. Pharmacol. Pharmacother. 1 (2010) 94-99.

[26] M.Y. Levy, S. Benita, Drug release from submicronized o/w emulsion: a new in vitro kinetic evaluation model, Int. J. Pharm. 66 (1990) 29-37.

[27] R. Lopes, C.V. Eleutério, L.M.D. Gonçalves, M.E.M. Cruz, A.J. Almeida, Lipid nanoparticles containing oryzalin for the treatment of leishmaniasis, Eur. J. Pharm. Sci. 45 (2012) 442-450.

[28] F. Maestrelli, P. Mura, M.J. Alonso, Formulation and characterization of triclosan submicron emulsions and nanocapsules, J. Microencaps. 21 (2004) 857-864.

[29] G. Mancini, R. Lopes, P. Clemente, S. Raposo, L. Gonçalves, A. Bica, H.M. Ribeiro, A.J. Almeida, Lecithin and parabens play a crucial role in tripalmitin-based lipid nanoparticle stabilization throughout moist heat sterilization and freezedrying: physical stability of tripalmitin solid lipid nanoparticles, Eur. J. Lipid Sci. Technol. 117 (2015) 1947-1959.

[30] W. Mehnert, K. Mäder, Solid lipid nanoparticles: production, characterization and applications, Adv. Drug Deliv. Rev. 64 (2012) 83-101.

[31] R.H. Muller, K. Mader, S. Gohla, Solid lipid nanoparticles (SLN) for controlled drug delivery: a review of the state of the art, Eur. J. Pharm. Biopharm. 50 (2000) 161-177.

[32] R.H. Muller, S. Runge, V. Ravelli, W. Mehnert, A.F. Thunemann, E.B. Souto, Oral bioavailability of cyclosporine: solid lipid nanoparticles (SLN) versus drug nanocrystals, Int. J. Pharm. 317 (1) (2006) 82-89.

[33] P. Mura, M. Valleri, M. Cirri, N. Mennini, New solid self-microemulsifying systems to enhance dissolution rate of poorly water soluble drugs, Pharm. Dev. Technol. 17 (3) (2012) 277-284.

[34] G. Neugebauer, G. Betzien, V. Hrstka, B. Kaufmann, E. von Mollendorff, U. Abshagen, Absolute bioavailability and bioequivalence of glibenclamide, Int. J. Clin. Pharmacol. Ther. Toxicol. 23 (1985) 453-460.

[35] P.O. Nnamani, A.A. Attama, E.C. Ibezim, M.U. Adikwu, SRMS142-based solid lipid microparticles: application in oral delivery of glibenclamide to diabetic rats, Eur. J. Pharm. Biopharm. 76 (2010) 68-74.

[36] O.J. Owolabi, E.K. Omogbai, Evaluation of the potassium channel activator levcromakalim (BRL38227) on the lipid profile, electrolytes and blood glucose levels of streptozotocin-diabetic rats, J. Diabetes. 5 (2013) 88-94.

[37] R. Paliwal, S. Rai, B. Vaidya, K. Khatri, A.K. Goyal, N. Mishra, A. Mehta, S.P. Vyas, Effect of lipid core material on characteristics of solid lipid nanoparticles designed for oral lymphatic delivery, Nanomedicine 5 (2) (2009) 184-191.

[38] D. Pandita, S. Kumar, N. Poonia, V. Lather, Solid lipid nanoparticles enhance oral bioavailability of resveratrol, a natural polyphenol, Food Res. Int. 62 (2014) 1165-1174.

[39] C.J. Porter, N.L. Trevaskis, W.N. Charman, Lipids and lipid-based formulations: optimizing the oral delivery of lipophilic drugs, Nat. Rev. Drug Discov. 6 (3) (2007) 231-248.

[40] C.J.H. Porter, C.W. Pouton, J.F. Cuine, W.N. Charman, Enhancing intestinal drug solubilisation using lipid-based delivery systems, Adv. Drug Deliv. Rev. 60 (2008) 673-691.

[41] C. Qi, Y. Chen, Q.Z. Jing, X.G. Wang, Preparation and characterization of catalase-loaded solid lipid nanoparticles protecting enzyme against proteolysis, Int. J. Mol. Sci. 12 (2011) 4282-4293.

[42] J. Qi, Y. Lu, W. Wu, Absorption, Disposition and pharmacokinetics of solid lipid nanoparticles, Curr. Drug Metab. 13 (2012) 418-428.

[43] Rohan M. Shah, François Malherbe, Daniel Eldridge, Enzo A. Palombo, Ian H. Harding, Physicochemical characterization of solid lipid nanoparticles (SLNs) prepared by a novel microemulsion technique, J. Colloid Interface Sci. 428 (2014) 286-294.

[44] E. Roger, F. Lagarce, J.P. Benoit, The gastrointestinal stability of lipid nanocapsules, Int. J. Pharm. 379 (2009) 260-265.

[45] B. Sanjula, F.M. Shah, A. Javed, A. Alka, Effect of poloxamer 188 on lymphatic uptake of carvedilol-loaded solid lipid nanoparticles for bioavailability enhancement, J. Drug Target. 17 (3) (2009) 249-256.

[46] C. Schwarz, W. Mehnert, Freeze-drying of drug-free and drug-loaded solid lipid nanoparticles, Int. J. Pharm. 157 (1997) 171-179.

[47] S.R. Shah, R.H. Parikh, J.R. Chavda, N.R. Sheth, Application of Plackett-Burman screening design for preparing glibenclamide nanoparticles for dissolution enhancement, Powder Technol. 235 (2013) 405-411.

[48] A.C. Silva, E. González-Mira, M.L. García, M.A. Egea, J. Fonseca, R. Silva, D. Santos, E.B. Souto, D. Ferreira, Preparation, characterization and biocompatibility studies on risperidone-loaded solid lipid nanoparticles (SLN): high pressure homogenization versus ultrasound, Colloids Surf. B 86 (1) (2011) 158-165.

[49] A.C. Silva, A. Kumar, W. Wild, D. Ferreira, D. Santos, B. Forbes, Long-term stability, biocompatibility and oral delivery potential of risperidone-loaded solid lipid nanoparticles, Int. J. Pharm. 436 (2012) 798-805.

[50] E.B. Souto, R.H. Müller, Lipid nanoparticles (SLN and NLC) for drug delivery, Handb. Exp. Pharmacol. 197 (2007) 115-141.

[51] M. Tobìo, A. Sànchez, A. Vila, I. Soriano, C. Evora, J.L. Vila-Jato, M.J. Alonso, The role of PEG on the stability in digestive fluids and in vivo fate of PEG-PLA nanoparticles following oral administration, Colloids Surf. B Biointerfaces 18 (2000) 315-323.

[52] B.M. Tashtoush, Z.S. Al-Qashi, N.M. Najib, In vitro and in vivo evaluation of glibenclamide in solid dispersion systems, Drug Dev. Ind. Pharm. 30 (2004) 601-607.

[53] M. Valleri, P. Mura, F. Maestrelli, M. Cirri, R. Ballerini, Development and evaluation of glyburide fast dissolving tablets using solid dispersion technique, Drug Dev. Ind. Pharm. 30 (2004) 525-534. 
[54] C. Vitorino, F.A. Carvalho, A.J. Almeida, J.J. Sousa, A.A.C.C. Pais, The size of solid lipid nanoparticles: an interpretation from experimental design, Colloids Surf. B. Biointerfaces 84 (2011) 117-130.

[55] H. Wei, R. Löbenberg, Biorelevant dissolution media as a predictive tool for glyburide a class II drug, Eur. J. Pharm. Sci. 29 (1) (2006) 45-52.

[56] S.A. Wissing, O. Kayser, R.H. Müller, Solid lipid nanoparticles for parenteral drug delivery, Adv. Drug Deliv. Rev. 56 (2004) 1257-1272.

[57] H. Yokokawa, I. Kinoshita, T. Hashiguchi, M. Kako, K. Sasaki, A. Tamura, Y. Kintaka, Y. Suzuki, N. Ishizuka, K. Arai, Y. Kasahara, M. Kishi, Y. Kobayashi, T. Takahashi, H. Shimizu, S. Inoue, Enhanced exercise-induced muscle damage and muscle protein degradation in streptozotocin-induced type-2 diabetic rats, J. Diabetes Investig. 2 (2011) 423-428.

[58] L. Yu, C. Li, Y. Le, J. Chen, H. Zou, Stabilized amorphous glibenclamide nanoparticles by high-gravity technique, Mater. Chem. Phys. 130 (2011) 361-366.
[59] H. Yuan, C.Y. Chen, G.H. Chai, Y.Z. Du, F.Q. Hu, Improved transport and absorption through gastrointestinal tract by PEGylated solid lipid nanoparticles, Mol. Pharm. 10 (2013) 1865-1873.

[60] M.G. Zariwala, N. Elsaid, T.L. Jackson, F. Corral López, S. Farnaud, S. Somavarapu, D. Renshaw, A novel approach to oral iron delivery using ferrous sulphate loaded solid lipid nanoparticles, Int. J. Pharm. 456 (2013) 400-407.

[61] N. Zerrouk, G. Corti, S. Ancillotti, F. Maestrelli, M. Cirri, P. Mura, Influence of cyclodextrins and chitosan, separately or in combination, on gliburide solubility and permeability, Eur. J. Pharm. Biopharm. 62 (3) (2006) 241-246.

[62] C.Y. Zhuang, N. Li, M. Wang, X.N. Zhang, W.S. Pan, J.J. Peng, Y.S. Pan, X. Tang, Preparation and characterization of vinpocetine loaded nanostructured lipid carriers (NLC) for improved oral bioavailability, Int. J. Pharm. 394 (1-2) (2010) 179-185. 